\title{
Gut leben zuhause mit Multimorbidität
}

\section{Praxisprojekt zum Beitrag der klinischen Pflegeexpertise MSc / APN in der häuslichen Pflege}

\author{
Rachel Jenkins 미, Anke Jähnke², Iren Bischofberger² \\ 'Spitex Zürich Limmat AG, Zürich \\ ${ }^{2}$ Careum Hochschule Gesundheit, Teil der Kalaidos Fachhochschule Schweiz, Zürich
}

\begin{abstract}
Zusammenfassung: Hintergrund: Immer mehr ältere Menschen leben mit Multimorbidität, reduzierter Autonomie und Lebensqualität, komplexen Therapieregimes und erhöhten Komplikations- und Hospitalisationsraten. Bei funktionalen Einschränkungen oder zur Unterstützung des Therapiemanagements ist die professionelle häusliche Pflege unerlässlich. In der stationären Versorgung verbessern Advanced Practice Nurses (APN) aufgrund ihrer erweiterten Kompetenzen die Versorgungsqualität. Für mehrfach erkrankte Klient_innen zuhause ist die Rolle der APN hingegen neu. Ziel: Ausgehend von international etablierten APN-Kernkompetenzen sollte die APN-Rolle in einem häuslichen Pflegedienst für Menschen mit Multimorbidität entwickelt werden. Methoden: Das Praxisprojekt orientierte sich am PDCA-Zyklus. Merkmale von zugewiesenen Klient_innen sowie Fallsituationen wurden analysiert und so der Mehrwert der APN für die Klient_innen, deren Angehörige, die Pflegeteams und die interprofessionelle Zusammenarbeit herausgearbeitet. Ergebnisse: Im Projektzeitraum wurden 40 Klient_ innen durch die APN begleitet. Durch ihre Interventionen stabilisierten sich vielschichtige Lebens- und Krankheitssituationen, und sie vermittelten den Beteiligten Sicherheit im Gesundheitsmanagement. Diskussion: Leadership in der Praxisentwicklung und interprofessionelle Zusammenarbeit waren zentrale Erfolgsfaktoren. Die APN nahm eine wichtige Koordinationsrolle innerhalb der zahlreichen Akteure wahr. Grenzen und Transfer: Diese Rolle muss für die häusliche Pflege konsolidiert und ihre Wirksamkeit auf die Versorgungsqualität empirisch erforscht werden.
\end{abstract}

Schlüsselwörter: Multimorbidität, alte Menschen, professionelle Pflege zuhause, Advanced Practice Nursing, evidenzbasierte Pflege

\section{Living well at home with multimorbidity - A project on the contribution of advanced practice nursing in home health care}

Abstract: Background: Multimorbidity has increased among the elderly, leading to loss of autonomy, lower quality of life, complex treatment plans and higher rates of complications and hospitalisations. Functional impairment and challenging therapy management make the use of home health nursing services essential. Experience in primary care and in hospitals has shown that Advanced Practice Nurses (APN) lead to a better quality of care for patients with multimorbidity. However, there is no data yet regarding the potential contribution of APNs to the care of these patients in home healthcare settings. Aim: To develop the role of the APN in a home health nursing organisation for patients with multimorbidity, applying internationally established APN core competencies. Methods: Characteristics of referred clients were collected and presented in case studies in an APN practice development project based on the PDCA-cycle. Benefits for clients, family caregivers, the nursing team, and for interprofessional collaboration were elaborated. Results: During the project period, a total of 40 clients were assigned to APN-care. An increase in stability in complex situations and higher confidence of clients and family caregivers in their individual health management were achieved. Discussion: The key factors were the APN's leadership role in best practice development and interprofessional collaboration. The APN played an important role in coordinating the numerous parties involved. Limits and transfer: This role must be further established. Empirical research is required to show the effect on quality of care.

Keywords: Multimorbidity, the elderly, home health nursing, Advanced Practice Nursing, evidence-based nursing

\section{Einleitung}

Die nachhaltige, bedarfsorientierte Versorgung von mehrfach erkrankten Menschen ist für die professionelle häusliche Pflege höchst anspruchsvoll. Das vorliegende Praxisprojekt beschreibt die Einführung von Pflegeexpert_innen
MSc/Advanced Practice Nurses (APN), um die vielfältigen Anforderungen dieser komplexen Pflegesituationen besser zu meistern.

Die Bewältigung von Multimorbidität ist international eine der wichtigsten Aufgaben in der Gesundheitsversorgung. Denn von Multimorbidität - d.h. dem gleichzeitigen 
Was ist zu dieser Thematik schon bekannt?

Advanced Practice Nurses (APN) verbessern die Pflegequalität bei mehrfach erkrankten Menschen in der stationären Versorgung.

\section{Was ist neu?}

Die APN stabilisiert in der häuslichen Pflege komplexe Lebens- und Krankheitssituationen und verbessert das Gesundheitsmanagement des Pflegeteams durch Leadership

Welche Konsequenzen haben die Ergebnisse für die Pflegepraxis?

Diese Rolle muss für die häusliche Pflege konsolidiert und ihre Wirksamkeit empirisch erforscht werden.

Auftreten von zwei oder mehr dauerhaften Gesundheitsbeeinträchtigungen und Symptomkomplexen (NICE, 2016) - sind in der Schweiz rund 22\% der über 50-Jährigen betroffen (Battegay, 2014). Der Anteil steigt ab 80 Jahren auf $41 \%$ (Moreau-Gruet, 2013) und wird sich bei dieser Altersgruppe bis 2045 verdoppeln (Weber et al., 2016). Herausfordernd ist nicht allein die Zahl der Gesundheitseinschränkungen, sondern deren Wechselwirkung im Kontext von Alter und Lebenssituation, die zu erhöhter Komplexität und Krankheitslast führen. Besonders stark betroffen sind alleinlebende Menschen (Haslbeck, Klein, Bischofberger \& Sottas, 2015), denn für sie intensivieren sich die Bewältigungsanforderungen. Die fragmentierte und spezialisierte Gesundheitsversorgung ist nicht auf diese Situationen ausgerichtet, denn diese erfordern ein Zusammenspiel von zahlreichen generalistischen und spezialisierten Diagnostik- und Therapieregimes. Durch den fehlenden Gesamtblick erhöhen sich das Komplikationsrisiko und die Hospitalisationsrate (Battegay, 2014; Moreau-Gruet, 2013).

\section{Hintergrund}

Als Grundlage für den Umgang mit Multimorbidität in der Primärversorgung dient oft das Chronic Care Modell (CCM) von Bodenheimer, Wagner \& Krumbach (2002), welches ein mehrdimensionales Vorgehen empfiehlt: interprofessionelles Team, Selbstmanagementförderung, Therapieentscheidungen nach Best-Practice-Vorgaben, Einbettung in gemeindenahe Unterstützungsangebote sowie elektronischer Informationsaustausch. Ergänzend zum CCM und um die Komplexität von Multimorbidität noch besser zu berücksichtigen, wurden klinische Leitfäden und Erkenntnisse zu diesem spezifischen Themenkomplex in der Primärversorgung hinzugezogen. Dazu gibt es inzwischen evidenzbasierte Strategien zur Priorisierung im Therapie- und Gesundheitsmanagement (American Geriatrics Society, 2012) sowie Guidelines (Mercer, Furler, Moffat, Fischbacher-Smith \& Sanci, 2016; NICE, 2016) und eine systematische Übersichtsarbeit (Smith, Wallace, Dowd \& Fortin, 2017). Fünf Handlungsschwerpunkte sind demnach bei Multimorbidität zentral: (1) Prioritäten, Werte und Ziele der Patient_innen als Ent- scheidungsgrundlage für die Therapie, (2) einfach umsetzbare Therapieregimes, (3) individuelle Versorgungspläne, (4) eine designierte Fachperson mit generalistischem Wissen zur Versorgungskoordination, (5) Unterstützung der pflegenden Angehörigen und Einbindung von verfügbaren Ressourcen des Gemeinwesens. Diese Handlungsschwerpunkte werden den Anforderungen der Praxissituationen erfahrungsgestützt gerechter, denn sie berücksichtigen sowohl die Behandlung als auch die personzentrierte Umsetzung im sozialen Kontext.

\section{Pflegeexpertise MSc / Advanced Practice Nurses}

In diesem Kontext bietet die konsekutive Masterausbildung in Pflege $(\mathrm{MScN})$ mit klinischem Schwerpunkt national (De Geest et al., 2008) und international (Schober et al., 2020) einen Mehrwert für die Gesundheitsversorgung. Neben erweiterten Untersuchungs- und Behandlungskompetenzen sowie der Verordnungsbefugnis (je nach örtlicher Gesetzgebung) sind MSc in Pflege Absolvent_innen als APN in der klinischen Praxis, Gesundheitsförderung, Patientenedukation und Koordination der interprofessionellen Zusammenarbeit tätig (Donald et al., 2013; Grant, Lines, Darbyshire \& Parry, 2017). In der medizinischen Grundversorgung (Laurant et al., 2018) und der stationären Langzeitpflege (Donald et al., 2013) ist diese Rolle international etabliert, und Qualität und Sicherheit der Leistungen sind unbestritten. In Deutschland, Österreich und der Schweiz sprachen sich die nationalen Berufsverbände der Pflegeberufe in einem gemeinsamen Positionspapier zur Pflegeexpertise MSc /APN auch in der Grundversorgung aus (DBfK, ÖGKV \& SBK, 2013). In Deutschland wurde die Community Health Nurse mit MSc-Abschluss für Gesundheitszentren eingeführt (Weskamm, Keßler \& Marks, 2018). In der Schweiz wird die Rolle zunehmend in der medizinischen Grundversorgung etabliert (Sailer Schramm et al., 2019).

Der zusätzliche Nutzen einer APN für Patient_innen mit Multimorbidität und ihre Angehörigen gründet auf ihrer zentralen Kompetenz der fortgeschrittenen klinischen Praxis und folgenden sechs Kernkompetenzen: Beratung und Coaching für Klient_innen und Angehörige, Konsultation und Unterstützung von Fachpersonen und Teams, intra- und interprofessionelle Zusammenarbeit, klinisches und fachliches Leadership, Implementation evidenzbasierter Praxis und ethische Entscheidungsfindung (Tracy \& O'Grady, 2019).

\section{Kontext Privathaushalt}

Die meisten Menschen mit Multimorbidität leben in Privathaushalten. Hier sind Hausarztpraxen (Herzig et al., 2018) und vor allem bei funktionalen Einschränkungen, Überbeanspruchung der Angehörigen oder Anforderungen im Therapiemanagement auch die professionelle häusliche Pflege (in der Schweiz auch Spitex genannt) ge- 
fragt. Dies trifft besonders - aber nicht nur - auf das hohe Alter zu. Im Jahr 2019 bezogen in der Schweiz 29\% der zuhause lebenden Personen ab 80 Jahren Spitexleistungen (Francis \& Lazzeri, 2020). Seit einigen Jahren beauftragen Spitexbetriebe Pflegeexpert_innen MSc/APN damit, die mehrfach erkrankten Klient_innen mit dem nötigen Rundumblick und Know-how zu unterstützen. Dies erfordert APNs mit generalistischen Kompetenzen, denn breit gefasste Aufgaben sind der größte Teil der Spitexleistungen (Imhof, de Wolf-Linder, Waldboth, Braun \& Mahrer Imhof, 2017), doch bislang waren APNs meist in Spezialgebieten wie palliativer oder psychosozialer Pflege tätig. Der MSc-Abschluss in Pflege ist in der Schweiz bisher weder im Gesundheitsberufegesetz geregelt, noch bestehen auf nationaler Ebene dafür vorgesehene Abrechnungsmöglichkeiten im Rahmen des Krankenversicherungsgesetzes. Diese regulatorische Situation eröffnet Betrieben und Kantonen ein Experimentierfeld für die Implementation fortgeschrittener Pflegepraxis in der Spitex, wobei dies jedoch lokal in der Ausgestaltung der Rolle und des Stellenprofils bei der Umsetzung verunsichern kann.

\section{Zielsetzung}

Ziel des Praxisprojekts war, die Funktion von generalistisch tätigen Pflegeexpert_innen MSc/APN in einem urbanen Spitexbetrieb der Deutschschweiz mit kommunalem Leistungsauftrag und Versorgungspflicht systematisch einzuführen und die Zusammenarbeit mit ausgewählten Spitexteams sowie der Hausärzteschaft im Hinblick auf eine „Gute APN-Praxis“ zu analysieren. In diesem Artikel fokussieren wir auf den APN-Beitrag zugunsten von Klient innen, Angehörigen und des Spitexbetriebs.

\section{Methode}

Methodisch orientierte sich das Praxisprojekt am PDCAZyklus nach Deming mit den Schritten „Planung - Umsetzung - Überprüfung - Anpassung" (Angermeier, 2016), die den Veränderungsprozess leiteten. Das Projekt war in das MScN-Studium der Erstautorin eingebettet und wurde, begleitet von den beiden Co-Autorinnen, von Januar 2016 bis Oktober 2019 durchgeführt.

Dieser Abschnitt konkretisiert die Umsetzung der einzelnen Schritte im Projektverlauf anhand des PDCAZyklus (siehe Abb. 1):

1. Planung: Erkennen von Verbesserungspotenzial, Definition eines Veränderungszieles und Planen von entsprechenden Maßnahmen.

2. Umsetzung: Testen der Maßnahmen. Wichtig ist, dass diese noch nicht etabliert werden, sondern dass eine Reaktion des Systems ausgelöst wird.

3. Überprüfung: Analyse der Auswirkungen. Die Ergebnisse entscheiden darüber, wie die Maßnahmen modifiziert und weiter umgesetzt werden.

4. Anpassung: Änderungen werden nachhaltig umgesetzt, z.B. indem neue Standards definiert werden. Um die positive Umsetzung voranzutreiben, setzt im Zyklus ein erneuter Schritt der Planung ein.

\begin{tabular}{|c|c|}
\hline $\begin{array}{l}\text { 01/2016-10/2016 } \\
\text { - Literaturgestützte Formulierung von } \\
\text { Aufgabenschwerpunkten in der } \\
\text { professionellen häuslichen Pflege bei } \\
\text { Menschen mit Multimorbidität } \\
\text { - Ausloten der betrieblichen } \\
\text { Integrationsmöglichkeiten der APN } \\
\text { - Formulierung von Überweisungskriterien an } \\
\text { die APN }\end{array}$ & $\begin{array}{l}\text { 11/2016-11/2018 } \\
\text { - Gezielte Information über Unterstützungsangebot } \\
\text { und Überweisungskriterien } \\
\text { - Beratung von Klient_innen und Angehörigen } \\
\text { - Einsatz erweiterter klinischer Kompetenzen } \\
\text { (Clinical Assessment) } \\
\text { - Personenbezogene Zusammenarbeit mit } \\
\text { Hausarztpraxen } \\
\text { - Edukation von Spitexteams } \\
\text { - Dokumentation aller Aktivitäten }\end{array}$ \\
\hline $\begin{array}{l}\text { 10/2019 bis zum aktuellen Zeitpunkt } \\
\text { - Vorschläge für die Anpassungen basierend } \\
\text { auf Erfahrungen des APN-Teams und } \\
\text { Rückmeldungen von Spitexteams } \\
\text { - Weitere Umsetzung und Schärfung der Rolle } \\
\text { - Erstellung eines betriebsinternen APN- } \\
\text { Konzeptes }\end{array}$ & $\begin{array}{l}\text { 11/2016-09/2019 } \\
\text { - Fortlaufende Analyse der Klientenmerkmale und } \\
\text { APN-Aktivitäten } \\
\text { - Regelmäßige Evaluation mit } \\
\text { Qualitätsbeauftragter im Betrieb } \\
\text { - Beratung und kollegialer Austausch im APN- } \\
\text { Team } \\
\text { - Selbstreflexion und Projekttagebuch } \\
\text { - Begleitende Reflexion und Coaching durch } \\
\text { MScN-Lehrkörper }\end{array}$ \\
\hline
\end{tabular}

Abbildung 1. Projektverlauf der APN-Rollenentwicklung in der Spitex für Menschen mit Multimorbidität und ihre Angehörigen. 


\section{Projektsetting}

Im Betrieb der Erstautorin (Anzahl Mitarbeitende zu Projektbeginn: 549 Vollzeitstellen, davon 3 APNs) sind seit 2014 Pflegeexpert_innen MSc/APN in verschiedenen Spezialgebieten wie palliativer oder psychosozialer Pflege tätig. Um den spezifischen Bedarf von mehrfach erkrankten Klient_innen in besonders komplexen Situationen zu decken, wurde 2016 die Stelle einer APN Chronic Care (im weiteren APN genannt) geschaffen und mit der Erstautorin besetzt. Ihr zur Seite standen die Qualitätsverantwortliche, eine betriebsinterne Projektgruppe und weitere APNs für den kollegialen Austausch.

In der Planungsphase erfolgte die Literaturanalyse $\mathrm{zu}$ Schwerpunkten der APN in der Spitex und ihrer Integration in den Betrieb. Gestützt darauf wurden Überweisungskriterien formuliert, anhand derer Klient_innen von Spitex-Mitarbeitenden und Hausarztpraxen an die APN überwiesen werden konnten. Dafür mussten zwei oder mehr chronische Erkrankungen vorliegen und mindestens eines der folgenden Kriterien zutreffen:

- Komplexes Therapie- und Medikamentenmanagement und Schwierigkeiten mit der Adhärenz

- Reduzierte Selbstmanagement-Fähigkeiten

- Notfälle und ungeplante Hospitalisationen wegen Exacerbation der Grunderkrankung

- Fehlendes, brüchiges oder stark belastetes soziales Netz

- Erschwerte Zusammenarbeit im interprofessionellen Team

- Anzeichen eines Frailty Syndroms im Alter

In einem nächsten Schritt stellte die APN das neue Angebot und die Überweisungskriterien in einem ausge- wählten Spitexzentrum (lokaler Stützpunkt für mehrere Spitexteams) sowie den organisationsinternen Fachstellen und einer hausärztlichen Gruppenpraxis vor. Diese überwiesen ihr im weiteren Projektverlauf Klient_innen. Sie übernahm die fachlich umfassende, evidenzbasierte und zeitlich intensive Begleitung, bis sich die gesundheitliche Situation so weit stabilisiert hatte, dass das örtliche Spitexteam die Fallführung übernehmen, resp. wieder übernehmen konnte. Die APN dokumentierte im Rahmen des Praxisprojekts kontinuierlich Klientenmerkmale, Probleme, Art und Dauer ihrer Unterstützung, bearbeitete Themen wie z.B. Therapiemanagement, die Mitwirkung pflegender Angehöriger oder die interprofessionelle Koordination und die aus ihrer Unterstützung resultierenden Situationsveränderungen. Sie erstellte jeweils personenbezogene Fallbeschreibungen und analysierte diese anhand der APN-Kompetenzen (Tracy \& O'Grady, 2019) mit dem Ziel, die Veränderungen durch die APN-Unterstützung zu identifizieren. Die Ergebnisse wurden zu unterschiedlichen Zeitpunkten im Rahmen des MScN-Studiums diskutiert und mit APN-Kolleginnen kritisch reflektiert.

Das Praxisprojekt fiel als betriebliche Qualitätsentwicklung ohne empirische Forschung nicht in den Geltungsbereich des Eidgenössischen Humanforschungsgesetzes, so dass keine Genehmigung durch die zuständige Ethikkommission erforderlich war. Die klinischen Daten der Dokumentation wurden anonymisiert und nach den geltenden Datenschutzvorgaben behandelt. Beide Personen, deren Daten die Grundlage der nachfolgenden Fallbeispiele bilden und deren Namen pseudonymisiert sind, willigten schriftlich für die Publikation gemäß den Empfehlungen von Schlüer et al. (2019) ein.

Tabelle 1. Merkmale der Klientensituationen

\begin{tabular}{|c|c|c|c|}
\hline Merkmal & Ausprägungen & Absolute Anzahl (n) & Prozentanteil (\%) \\
\hline \multirow[t]{2}{*}{ Geschlecht } & weiblich & 31 & 77 \\
\hline & männlich & 9 & 23 \\
\hline \multirow[t]{2}{*}{ Alter (in Jahren) } & Median: 80 & & \\
\hline & Altersspanne: 29 - 92 & & \\
\hline Wohnsituation & Alleine lebend & 21 & 52 \\
\hline \multirow{7}{*}{$\begin{array}{l}\text { Häufigste medizinische Diagnosen } \\
\text { ( } n=52, \text { Mehrfachnennungen möglich) }\end{array}$} & Psychiatrische Erkrankung & 12 & 23 \\
\hline & (davon Depression) & (7) & (13) \\
\hline & Chronisches Schmerzsyndrom & 10 & 19 \\
\hline & Demenzielle Entwicklung & 8 & 15 \\
\hline & Koronare Herzkrankheit & 8 & 15 \\
\hline & Muskuloskelettale Erkrankung & 7 & 14 \\
\hline & Malnutrition & 7 & 14 \\
\hline \multirow{4}{*}{$\begin{array}{l}\text { Häufigste Gründe für Unterstützungsbedarf } \\
\text { ( } n=43 \text {, Mehrfachnennungen möglich) }\end{array}$} & Symptomlast und Therapieanforderungen & 14 & 33 \\
\hline & Schmerzmanagement & 11 & 26 \\
\hline & Sturz / Sturzrisiko & 10 & 23 \\
\hline & Belastung pflegender Angehöriger & 8 & 19 \\
\hline \multirow[t]{2}{*}{ Anzahl verordneter Arzneimittel } & $\geq 15$ verordnete Arzneimittel & 5 & 12,5 \\
\hline & $10-14$ verordnete Arzneimittel & 8 & 20 \\
\hline (Anzahl wird nur von Klient_innen erfasst, bei & $5-9$ verordnete Arzneimittel & 6 & 15 \\
\hline denen die Spitex das Medikamentenmanagement & $<5$ verordnete Arzneimittel & 2 & 5 \\
\hline übernimmt, d.h. hier: nur bei 21 der 40 Klient_innen) & Nicht bekannt & 19 & 47,5 \\
\hline
\end{tabular}




\section{Ergebnisse}

Im Projektzeitraum wurden der APN insgesamt 40 Klient innen überwiesen (von Spitexzentrum und -fachteams sowie Hausarztpraxis), wobei die Gesamtzahl an Klient_innen des zuweisenden Spitexzentrums und der Fachteams im selben Zeitraum nicht ermittelt wurde. Tabelle 1 zeigt ausgewählte Merkmale der überwiesenen Klient_innen. Ihre soziodemographischen und krankheitsbezogenen Merkmale unterscheiden sich nicht wesentlich von der Gesamtpopulation der Klient_innen des Spitexbetriebs, d.h. hohes Alter und Multimorbidität sind dominante Merkmale bei Spitex-Leistungsbezüger_innen (Imhof et al., 2017).

Aus den 40 Situationen wählten wir zwei Beispiele mit komplementären Aspekten und besonders aussagekräftigen Veränderungen aus. Diese Beispiele illustrieren die angestrebte "Gute APN-Praxis“ in der zentralen Kompetenz der direkten klinischen Praxis wie auch in den sechs Kernkompetenzen. Ihre Analyse wird in Tabelle 2 anhand des zugrundliegenden Modells dargestellt.

\section{Fallbeispiel 1: Sicherheit durch beratende Begleitung}

Herr Dünki, 80-jährig, Hauptdiagnose progrediente Lungenerkrankung, Nebendiagnosen koronare Herzkrankheit und chronisches thorakales Schmerzsyndrom unklarer Ursache, leichte kognitive Einschränkung. Er wohnte zusammen mit seiner Ehefrau, die ihn im Selbstmanagement unterstützte. Seine Hausärztin überwies ihn zur Abklärung und Unterstützung an die APN mit dem Ziel, die Situation zu stabilisieren, sein Selbstmanagement zu fördern und die Versorgung vorauszuplanen. Die APN-Einsätze beim Ehepaar erfolgten während eines Jahres flexibel und angepasst an die jeweilige Verlaufsdynamik der Mehrfacherkrankungen mit den Schwerpunkten Selbstmanagementschulung in der Symptominterpretation und Therapieumsetzung. Dank vertieftem klinischen Assessment, erweiterten pharmakologischen Kenntnissen und kontinuierlicher, zeitnaher Zusammenarbeit mit der Hausärztin verringerte sich die Symptomlast und verbesserte sich die Lebensqualität deutlich. Das Ehepaar resümierte den Einsatz der APN: „Es gibt eine enorme Sicherheit, dass man nicht allein auf sich gestellt ist, und dass man jemanden im Hintergrund hat, zu dem man Vertrauen hat."

\section{Fallbeispiel 2: Spitex statt Übergangspflege im Pflegezentrum}

Frau Tobler, 85-jährig, Austritt nach Hause direkt nach dem Spitalaufenthalt wegen Schenkelhalsfraktur, insulinpflichtiger Diabetes mit instabilen Blutzuckerwerten, Belastungsdyspnoe bei Herzinsuffizienz, starke Rückenschmerzen durch Wirbelkompressionsfrakturen bei Osteoporose, allein wohnend im 2. Stock ohne Lift, Tochter wohnte in einer anderen Gemeinde. Ziele der Unterstützung waren der kontinuierliche Mobilitätsaufbau und die funktionale Unabhängigkeit. Zuhause nach dem Spitalaustritt war Fr. Toblers erste Aussage: „Ich verlasse meine Wohnung jetzt ein halbes Jahr nicht mehr!" Nach vier Monaten erreichte sie allerdings dank den systematischen und konsequenten APN-Interventionen sowie der Unterstützung des Spitexteams eine bessere Mobilität und einen größeren Bewegungsradius als vor der Schenkelhalsfraktur. Dies illustriert ihre Aussage gegenüber der APN: „Ich kann jetzt nicht lange telefonieren, ich bin am Einkaufen.“

Die Analyse zeigt, dass die APN die beiden zunächst instabilen Fallsituationen mit intensiver Unterstützung und umsichtigem Gesundheitsmanagement so stabilisierte, dass keine weiteren Hospitalisationen wegen Exacerbation der Grunderkrankung oder Stürzen erfolgten. Das Ehepaar Dünki erhielt eine sorgfältige, individuell ausgerichtete Beratung und Edukation zu Symptombeobachtung, -interpretation und Therapie und erlangte so mehr Sicherheit im Selbstmanagement. Die APN arbeitete beim Spitalaustritt eng mit dem Spitalteam zusammen und gewährleistete so den sicheren Übergang nach Hause. Intensives Coaching in Mobilitätsaufbau, Ernährung und Therapiemanagement führten bald zu verbesserter funktioneller Gesundheit im Alltag.

In beiden Situationen gleiste die APN die engmaschige und proaktive Zusammenarbeit mit den Hausärztinnen auf. Dies bot dank zielgerichteter und konziser Kommunikation allen Beteiligten die nötige Orientierung im Prozess. Die APN vermittelte dem zuständigen Spitexteam spezifische Kenntnisse und Kompetenzen für die Situation von Frau Tobler und lebte bei gemeinsamen Einsätzen die personzentrierte Kommunikation mit der Kundin vor.

Aufgrund dieser Erkenntnisse integrierte der Spitexbetrieb ab Oktober 2019 die APN-Rolle für mehrfach erkrankte Klient_innen mit herausfordernden Altagssituationen in die Regelversorgung und weitete das Angebot auf den ganzen Betrieb aus. Siehe Absatz Ergebnisse, zweiter Paragraph.

\section{Diskussion}

Die kontinuierliche Reflexion und Analyse der Fallbeispiele aus dem Praxisprojekt zeigten, dass die „Gute APNPraxis“ drei maßgebliche Elemente umfasst: a) zweckmäßige Triage von Klient_innen, b) spezifischer Mehrwert der Rolle für Klient_innen, Angehörige und Spitexteams und c) die Versorgungskoordination im interprofessionellen Kontext.

\section{Triage von Klient_innen}

Eine APN erkennt aufgrund ihrer Pflegeexpertise frühzeitig die komplexen Zusammenhänge und Risiken im Versorgungsverlauf und baut rasch Problemlösungsstrate- 
Tabelle 2. Analyse der Fallbeispiele nach APN-Kompetenzen

\begin{tabular}{ll}
\hline APN-Kompetenz & Wesentlicher Beitrag der APN in den Fallsituationen \\
\hline Direkte klinische Praxis & In beiden Situationen führte das systematische klinische Assessment zu Befunden, die durch eine ärztliche \\
& Abklärung bestätigt wurden und in Therapieanpassungen resultierten. Bei beiden Personen wurde ein \\
& Medikamentenabgleich durchgeführt und ein funktionierendes Medikamentenmanagement eingeführt. \\
& Herr Dünki: Die APN erfasste beim kardiopulmonalen Assessment frühzeitig eine Exacerbation, sodass die \\
& notwendigen Schritte umgehend eingeleitet werden konnten und rasch zu einer Symptomverbesserung \\
& führten. \\
& Frau Tobler: Die APN auskultierte ein noch nicht dokumentiertes Strömungsgeräusch über der Aorten- \\
& klappe. Die anschließende kardiologische Abklärung mit Anpassung der Herzmedikamente reduzierte \\
& ihre störende Dyspnoe bei körperlicher Betätigung spürbar und verbesserte ihre Lebensqualität.
\end{tabular}

Beratung und Coaching Herr und Frau Dünki: Das Ehepaar benötigte Selbstmanagementförderung zur Therapieumsetzung sowie Anleitung bei der Symptombeobachtung und -interpretation. Dazu wurden ein für das Ehepaar gut verständliches und umsetzbares Symptomtagebuch und ein Maßnahmenschema mit Ampelsystem eingeführt. Die APN besprach bei den regelmäßigen Einsätzen den Verlauf anhand der Tagebucheinträge und reflektierte Verhaltensmaßnahmen.

Frau Tobler: Die Klientin brauchte Unterstützung, um ihre Mobilität außer Haus wiederzuerlangen. Dank motivierender Gesprächsführung konnte sie ihre Prioritäten formulieren und für sie realistische und sinnvolle Ziele setzen. Die APN beriet sie erfolgreich, wie sie ihren Bewegungsradius vergrößern, ihre Beweglichkeit aufbauen und eine passende Turngruppe im Quartier finden konnte.

Konsultation

Frau Tobler: Das Spitexteam war maximal gefordert durch den Spitalaustritt nach Schenkelhalsoperation direkt nach Hause, und nicht wie üblich zunächst in eine Rehabilitationsklinik. Erschwerend waren die instabilen somatischen Erkrankungen. Die APN begleitete die fallführende Pflegefachfrau beim ersten Abklärungseinsatz, unterstützte sie engmaschig im Aufbau eines stabilen Pflegearrangements sowie der Dokumentation von Wirksamkeit und Zweckmäßigkeit der Pflegeleistungen. Das Team wurde in der Mobilitätsförderung spezifisch zur Situation von Frau Tobler geschult. Psychosoziale Herausforderungen wurden in einer Fallbesprechung bearbeitet.

Intra- und interprofessionelle Zusammenarbeit

Die APN gewährleistete den Informationsfluss zwischen den verschiedenen involvierten Gesundheitsfachpersonen und koordinierte deren Einzelleistungen.

Herr Dünki: Der regelmäßige, datengestützte E-Mail-Kontakt mit der Hausärztin ermöglichte einen nahtlosen Informationsfluss.

Frau Tobler: Um die Sicherheit beim Übertritt nach Hause zu gewährleisten, besuchte die APN Frau Tobler bereits im Akutspital und beschaffte sich vom Spitalteam Informationen. So konnte sie die Austrittsvorbereitung beeinflussen, z. B. die Einstellung der schwankenden Blutzuckerwerte. Da diese zuhause anhielten, organisierte sie zeitnah eine Diabetesberatung, wonach die Werte sich deutlich verbesserten. Frau Tobler gewann dadurch Selbstvertrauen im selbständigen Therapiemanagement.

Evidenz basierte Praxis In den Fallbeispielen wurde die Pflegepraxis anhand evidenzbasierter Interventionen laufend weiterentwickelt.

Herr Dünki: Ein betriebsinterner Standard für Klient_innen mit COPD wurde getestet und Instrumente wie das Symptomtagebuch angepasst.

Frau Tobler: Gemachte Erfahrungen flossen in die Leitfadenentwicklung zur Mobilitätsverbesserung ein.

Leadership Herr Dünki: Die APN gestaltete als Vorreiterin die beratende Rolle im Gesundheitsmanagement bei Multimorbidität.

Frau Tobler: Die APN nahm dank enger Zusammenarbeit mit dem Spitexteam eine Vorbildfunktion ein. In gemeinsamen Einsätzen vertieften die Pflegefachpersonen ihre Kompetenzen, und in der Fallbesprechung übten sie die Priorisierung in der komplexen Pflegesituation.

Ethische Entscheidungsfindung Frau Tobler: Durch den Spitalaustritt direkt nach Hause (ohne Zwischenaufenthalt in der Akut- und Übergangspflege oder einer Rehabilitationsklinik) zeigte sich das ethische Dilemma Autonomie versus Fürsorge. Das Spital- und Spitexteam sowie der Hausarzt schätzten unter Federführung der APN gemeinsam ein, ob die Sicherheit zuhause gewährleistet sei. Ein Konsens unter den Beteiligten zeigte, welche Bedingungen für einen Austritt erfüllt sein sollten.

gien sowie eine interprofessionelle Kollaboration auf (Tracy \& O'Grady, 2019). Ziel der Triage ist, mehrfach erkrankte Klient_innen in komplexen Situationen und instabilem Krankheitsverlauf zu erfassen. Die soziodemographischen und krankheitsbezogenen Merkmale der ersten 40 Klient_innen im APN-Projekt unterschieden sich nicht wesentlich von der Gesamtpopulation des Spitexbetriebs. Insofern sind die Überweisungskriterien für Spitexteams und Hausärzteschaft an die APN empirisch zu überprüfen und zu schärfen. Damit die APN ihre fortgeschrittenen Kompetenzen noch fundierter einsetzen kann, könnte sie krankheitsspezifische Kriterien der Klienten- situation proaktiv und risikobasiert einschätzen, um Belastungssituationen frühzeitig zu identifizieren.

\section{Spezifischer Mehrwert der Rolle}

Erkrankungen, Therapien, funktionale Einschränkungen und Lebenssituation beeinflussen sich in den Fallbeispielen gegenseitig. Priorisierungen im Krankheitsmanagement waren nötig, um Veränderungen rechtzeitig anzugehen und gleichzeitig den Möglichkeiten, Vorstellungen und Zielen von Klient_innen und Angehörigen gerecht zu 
werden (Mercer et al., 2016; NICE, 2016). Die Fähigkeit, den Alltag gemäß eigener Bedürfnisse und Wünsche zu gestalten und zu bewältigen, ist für die Lebensqualität im Alter bei Multimorbidität zentral (Weber et al., 2016). In den beiden Fallbeispielen war dies dank Alltagsnähe, kommunikativen Fähigkeiten und einem Rundum-Blick der APN auf die individuelle Situation möglich (Bonsack \& Reichart, 2013; Trilla et al., 2018).

Grundlage war das umfassende klinische Assessment durch die APN, mit welchem sie die Ausprägungen der Gesundheitseinschränkungen der Klient_innen erfasste und entsprechend proaktiv und partizipativ die Versorgung mit Klient_innen, Angehörigen und dem interprofessionellen Team plante (Counsell et al., 2007; Imhof, Naef, Wallhagen, Schwarz \& Mahrer Imhof, 2012). Die systematische Durchführung eines multidimensionalen geriatrischen Assessments ist im stationären Setting bereits etabliert. Für die professionelle Pflege zuhause ist dies, wie in einzelnen Studien ersichtlich, noch ausbaufähig (Pilotto \& Martin, 2018).

In beiden Fallbeispielen half die APN den Spitexteams, die Themen in den Konsultationen situativ zu priorisieren und so eine stabile Situation zu erreichen. Die Spitexteams profitierten dabei von ihren Leadership-Kompetenzen und der Vermittlung von evidenzbasiertem Wissen. Zudem konnten sie niederschwellige Inputs zur praktischen Umsetzung auf weitere Pflegesituationen übertragen. Die APN hatte dabei eine Vorbildfunktion und diente auch als Change Agent (Tracy \& O'Grady, 2019).

\section{Versorgungskoordination im Kontext interprofessioneller Zusammenarbeit}

Die APN hat dank ihres geschärften Blicks auf das Gesamtsystem der Gesundheitsversorgung einschließlich der Finanzierungsmechanismen eine auch international breit erforschte Funktion in der Versorgungskoordination bei Multimorbidität (Doessing \& Burau, 2015). Dadurch sinkt in einigen ambulanten Versorgungsmodellen die Häufigkeit von akuten Ereignissen mit Notfalleinweisung und Hospitalisation signifikant (Councell et al., 2007; Imhof et al., 2012; Trilla et al., 2018). Die Fallbeispiele verdeutlichen, wie die APN in ihrer Spitexpraxis durch klinische, fachliche und kommunikative Expertise maßgeblich zu Kontinuität, Zugang und Sicherheit in der Gesundheitsversorgung beitrug. Diese so ausgeübte Versorgungskoordination beugt Komplikationen vor. In der Schweiz ist die Koordination bereits heute als verrechenbare Leistung der professionellen häuslichen Pflege möglich, muss aber immer wieder argumentiert werden (Giger, Häusler, Sander \& Staffelbach, 2018).

\section{Grenzen}

Limitierend an diesem Praxisprojekt ist, dass die betriebliche Rolle stark kontextabhängig und daher nicht un- mittelbar auf andere Situationen übertragbar ist. Zudem hatte das Projekt Pilotcharakter, bei dem der PDCA-Zyklus den methodischen Rahmen bildete. Die Erhebung empirischer Daten stand bei der Planung und Durchführung nicht im Zentrum, so dass z. B. keine robusten Aussagen zu Outcomes möglich sind. Dennoch können die Ergebnisse Impulse für die professionelle häusliche Pflege geben, auch über die Landesgrenze hinweg, und ebenfalls für ein etwaiges empirisches Forschungprojekt.

\section{Schlussfolgerungen und Transfer}

Der geschärfte Blick auf die Lebenswelt, die Nutzung umfassender klinischer Kompetenzen, die gezielte interprofessionelle Kommunikation und die umsichtige Praxisentwicklung sind als Kern des Projekterfolgs zu werten. Um darüber hinaus das volle Potenzial der Kompetenzen von Pflegeexpert_innen MSc / APN für eine wirksame und effiziente häusliche Pflege auszuschöpfen, muss die Rolle auf individueller, betrieblicher und staatlicher Ebene weiter erprobt, entwickelt und deren Wirksamkeit durch Begleitforschung mit definierten Prozess- und Outcome-Indikatoren überprüft werden. Dadurch könnten sowohl die Versorgungs- als auch die Lebensqualität von Klient_innen und deren Angehörigen maßgeblich gefördert werden.

Die gesetzliche Reglementierung der Masterausbildung mit klinischem Fokus und die entsprechende Berufsausübung sowie die Finanzierung der Leistungen sollten zukünftig weiterentwickelt werden. Bis dahin können Betriebe in der Experimentalphase ihre APN-Aktivitäten evaluieren (lassen), um diese nachhaltig auszurichten. Dadurch wird gewährleistet, dass die Versorgungsqualität nicht einzelnen engagierten Spitexbetrieben und klinischen Pflegeexpert_innen MSc/APN oder dem Zufall überlassen bleibt, sondern sich auf eine solide Wissensbasis stützt und damit Chancengleichheit für Klient_innen und Angehörige ermöglicht.

\section{Literatur}

American Geriatrics Society. (2012). Patient-centered care for older adults with multiple chronic conditions: a stepwise approach from the American Geriatrics Society. Expert Panel on the Care of Older Adults with Multimorbidity. Journal of the American Geriatrics Society, 60(10), 1957 - 1968.

Angermeier, D. (2016). PDCA-Zyklus. Projektmagazin. Verfügbar unter https://www.projektmagazin.de/glossarterm/pdca-zyklus [13.06.2021].

Battegay, E. (2014). Multimorbidität: eine Herausforderung der Neuzeit. Schweizerische Akademie der Medizinischen Wissenschaften Bulletin, 14(4), 1- 4.

Bodenheimer, T., Wagner, E.H. \& Krumbach, K. (2002). Improving primary care for patients with chronic illness - The Chronic Care Model Part 2. Journal of the American Medical Association, 288(14), $1909-1914$.

Bonsack, S. \& Reichart C. (2013). Neue Rollen der Pflege in der erweiterten Hausarztmedizin. leila Leben mit Langzeiterkrankung. Stadt Zürich. Städtische Gesundheitsdienste. Verfügbar unter https://gn2025.ch/wp-content/uploads/2018/05/LEILA_ 
Neue_Rollen_der_Pflege_in_der_erweiterten_Hausarztmedizin _2013_web.pdf [20.09.2021].

Counsell, S.R., Callahan, C.M., Clark, D.O., Tu, W., Buttar, A.B., Stump, T.E. et al. (2007). Geriatric care management for lowincome seniors: a randomized controlled trial. Journal of the American Medical Association, 298(22), 2623-2633.

De Geest, S., Moons, P., Callen, B., Gut, C., Lindpaintner, L.S. \& Spirig, R. (2008). Introducing Advanced Practice Nurses/ Nurse Practitioners in health care systems: a framework for reflection and analysis. Swiss Medical Weekly, 138(43 - 44), $621-628$.

Deutscher Berufsverband für Pflegeberufe (DBfK); Österreichischer Gesundheits- und Krankenpflegeverband (ÖGKV) \& Schweizer Berufsverband der Pflegefachfrauen und Pflegefachmänner (SBK - ASI). (2013). Advanced Nursing Practice in Deutschland, Österreich und der Schweiz. Eine Positionierung von DBfK, ÖGKV und SBK. Verfügbar unter https://www.dbfk.de/media/docs/ download/DBfK-Positionen/ANP-DBfK-OeGKV-SBK_2013.pdf [13.06.2021].

Doessing, A. \& Burau, V. (2015). Care coordination of multimorbidity: a scoping study. Journal of Comorbidity, 5, $15-28$.

Donald, F., Martin-Misener, R., Carter, N., Donald, E., Kaasalainen, S., Wickson-Griffiths, A. et al. (2013). A systematic review of the effectiveness of advanced practice nurses in long-term care. Journal of Advanced Nursing, 69(10), 2148-2161.

Francis, A. \& Lazzeri, F. (2020). Medienmitteilung. Sozialmedizinische Betreuung in Institutionen und zu Hause 2019. Bundesamt für Statistik BFS, Espace de l'Europe 10, Neuchâtel. Verfügbar unter https://www.bfs.admin.ch/bfs/de/home/aktuell/neueveroeffentlichungen.assetdetail.1817268.html [20.09.2021].

Giger, M.-A., Häusler, E., Sander, M. \& Staffelbach, D. (2018). Abgeltung von Leistungen im Rahmen der koordinierten Versorgung. Bern: Bundesamt für Gesundheit.

Grant, J., Lines, L., Darbyshire, P. \& Parry, Y. (2017). How do nurse practitioners work in primary health care settings? A scoping review. International Journal of Nursing Studies, 75, 51 - 57.

Haslbeck, J., Klein, M., Bischofberger, I. \& Sottas, B. (2015). Leben mit chronischer Krankheit. Die Perspektive von Patientinnen, Patienten und Angehörigen. Obsan Dossier 46. Neuchâtel: Schweizerisches Gesundheitsobservatorium.

Herzig, L., Mueller, Y., Zeller, A., Streit, S., Haller, D., Neuner-Jehle, S. et al. (2018). Das Programm Multimorbidität in der Hausarztmedizin. Schweizerische Ärztezeitung, 2018.06949. Verfügbar unter https: //saez.ch/de/article/doi/saez.2018.06949/ [13.06.2021].

Imhof, L., De Wolf-Linder, S., Waldboth, V., Braun, A. \& Mahrer Imhof, R. (2017). Vergleich von Patientengruppen mit einem hohen und tiefen Versorgungsgrad in der Spitex Zürich Sihl. Unveröffentlichte Arbeit, Zürcher Hochschule für Angewandte Wissenschaften.

Imhof, L., Naef, R., Wallhagen, M.I., Schwarz, J. \& Mahrer Imhof, R. (2012). Effects of an advanced practice nurse in-home health consultation program for community-dwelling persons aged 80 and older. Journal of the American Geriatrics Society, 60(12), $2223-2231$

Laurant, M., van der Biezen, M., Wijers, N., Watananirun, K., Kontopantelis, E. \& van Vught AJAH. (2018). Nurses as substitutes for doctors in primary care. Cochrane Database of Systematic Reviews, 7, CD001271.

Mercer, S., Furler, J., Moffat, K., Fischbacher-Smith, D. \& Sanci, L. (2016). Multimorbidity. Technical Series on Safer Primary Care. Geneva: World Health Organization.

Moreau-Gruet, F. (2013). Multimorbidität bei Personen ab 50 Jahren. Ergebnisse der Befragung SHARE (Survey of Health, Ageing and Retirement in Europe). Obsan Bulletin, 4/2013. Neuchâtel: Schweizerisches Gesundheitsobservatorium.

National Institute for Health and Care Excellence (NICE). (2016). Multimorbidity: Clinical Assessment and Management. Verfügbar unter https://www.nice.org.uk/guidance/ng56 [13.06.2021].

Pilotto, A. \& Martin, F.C. (2018). Comprehensive Geriatric Assessment. Cham: Springer International Publishing.
Sailer Schramm, M., Brüngger, B., Wyss, C., Röthlisberger, A., Kläy, M., Triaca, H. et al. (2019). Tandembetreuung mit Vorteilen für alle Beteiligten. Patientenkonferenzen verdeutlichen den Kompetenzenschift. Primary and Hospital Care - Allgemeine innere Medizin, 19(2), $52-56$.

Schlüer, A., Schori D., Rettke H., Sauer R., Kuster B., Gemperle. R. et al. (2019). Datenschutz und Datensicherheit bei der Durchführung von Studien und Praxisentwicklungsprojekten in der Pflege. Ein gemeinsames Vorgehen in den vier universitären Spitälern der Stadt Zürich. Pflegerecht, 2/ 2019, 91 - 93.

Schober, M., Lehwaldt, D., Rogers, M., Steinke, M., Turale, S., Pulcini, J. et al. (2020). Guidelines on Advanced Practice Nursing. International council of Nursing (ICN). Geneva. Verfügbar unter https: //www.icn.ch/system/files/documents/2020-04/ICN_APN\%20 Report_EN_WEB.pdf [13.06.2021].

Smith, S.M., Wallace, E., O'Dowd, T. \& Fortin, M. (2016). Interventions for improving outcomes in patients with multimorbidity in primary care and community settings. Review. Cochrane Database Syst. Rev., 3, CD006560.

Tracy, M.F. \& O'Grady, E.T. (eds.). (2019). Hamric and Hanson's Advanced Practice Nursing. An Integrative Approach (6th ed.). St. Louis: Saunders/Elsevier.

Trilla, F., DeCastro, T., Harrison, N., Mowry, D., Croke, A., Bicket, M. et al. (2018). Nurse practitioner home-based primary care program improves patient outcomes. Journal for Nurse Practitioners, 14(9), e185-e188.

Weber, D., Abel, B., Ackermann, G., Biedermann, A., Bürgi, F., Kessler, C. et al. (2016). Gesundheit und Lebensqualität im Alter. Grundlagen für das kantonale Aktionsprogramm „Gesundheitsförderung im Alter". Gesundheitsförderung Schweiz Bericht 5, Bern und Lausanne. Verfügbar unter GFCH_2016_Gesundheit_ und_Lebensqualitaet_im_Alter.pdf (npg-rsp.ch) [20.09.2021].

Weskamm, A., Keßler, I.G. \& Marks, F. (Hrsg.). (2018). Community Health Nursing in Deutschland. Konzeptionelle Ansatzpunkte für Berufsbild und Curriculum. Agnes-Karll-Gesellschaft für Gesundheitsbildung und Pflegeforschung $\mathrm{mbH}$, vertreten durch den Deutschen Berufsverband für Pflegeberufe - DBfK Bundesverband e.V., Berlin. Verfügbar unter www.dbfk.de/media/ docs/Bundesverband/CHN-Veroeffentlichung/BroschuereCommunity-Health-Nursing-09-2019.pdf [20.09.2021].

\section{Historie}

Manuskripteingang: 15.12.2020

Manuskript angenommen: 27.09.2021

Onlineveröffentlichung: 07.10.2021

\section{Autorenschaft}

Substanzieller Beitrag zu Konzeption oder Design der Arbeit: $\mathrm{RJ}, \mathrm{AJ}, \mathrm{IB}$

Substanzieller Beitrag zur Erfassung, Analyse oder Interpretation der Daten: RJ, AJ

Manuskripterstellung: RJ, AJ, IB

Einschlägige kritische Überarbeitung des Manuskripts: RJ, AJ, IB Genehmigung der letzten Version des Manuskripts: RJ, AJ, IB Übernahme der Verantwortung für das gesamte Manuskript: RJ, AJ, IB

\section{Danksagung}

Ein herzlicher Dank geht an Arda Teunissen, Pflegeleitung Spitex Zürich Limmat, und die Teamkolleginnen der Fachentwicklung Chronic Care: Nicole Hollenstein, Sandra Kunkel,

Azra Karabegovic und Christine Reichart.

\section{ORCID}

Rachel Jenkins

(D) https://orcid.org/0000-0003-0443-0916 


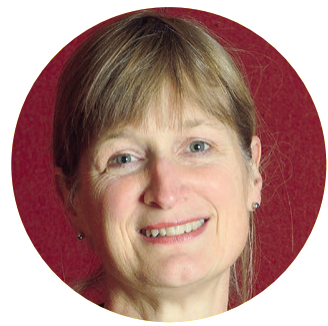

Rachel Jenkins, RN, MScN, MPH

Spitex Zürich Limmat AG

Rotbuchstrasse 46

8037 Zürich

Schweiz

rachel.jenkins@spitex-zuerich.ch

Was war die größte Herausforderung bei Ihrem Praxisprojekt? Die Projektdurchführung in einem sehr dynamischen beruflichen und betrieblichen Umfeld.

\section{Was wünschen Sie sich bezüglich der Thematik für die Zukunft?} Eine bereichernde Zusammenarbeit unter APN Kolleg_innen verschiedener Pflegesettings, damit die Rolle an Ausstrahlungskraft und Impact gewinnt.

\section{Was empfehlen Sie zum Weiterlesen/Vertiefen?}

Tracy, M.F. \& O'Grady, E.T. (eds.). (2019). Hamric and Hanson's Advanced Practice Nursing. An Integrative Approach (6th ed.). Siehe Literatur.
Anzeige

\section{Ursache und Wirkung verstehen}

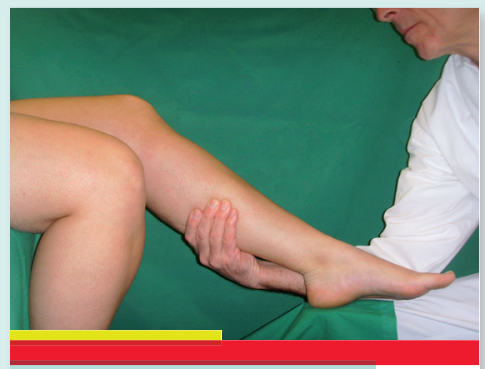

\section{Phlebologie} Paradigmenwechsel

(1) hogrefe

Thomas Stumptner

\section{Phlebologie}

Plädoyer für einen überfälligen Paradigmenwechsel

2021. 180 S., 59 Abb., Kt

$€ 34,95$ (DE) / € 36,00 (AT) / CHF 47.90

ISBN 978-3-456-86050-3

Auch als eBook erhältlich

Dieses Buch soll das Verständnis für eine umfassende Behandlung phlebologischer Erkrankungen (Venenstau, Beinekzem oder auch offenes Bein) und möglichen Folgeerkrankungen wie Beinvenenthrombose, Krampfadern mit seinen Ursachen und Therapieoptionen ermöglichen und fördern. Das Buch richtet sich v.a. an alle, die am alltäglichen Versorgungsprozess von Patienten mit einer Kompressionstherapie beteiligt sind.

www.hogrefe.com 